\title{
Twinning and Risk of Stillbirth Subtypes in Pediatric Mothers
}

\author{
Hamisu M. Salihu, ' Puza P. Sharma, ${ }^{2}$ and Shillena Peters ${ }^{\prime}$ \\ ' Department of Obstetrics, Gynecology, and Reproductive Sciences, UMDNJ-Robert Wood Johnson Medical School, New Brunswick, New Jersey, \\ United States of America \\ ${ }^{2}$ Department of Epidemiology, UMDNJ-School of Public Health, New Jersey, United States of America
}

W e sought to estimate levels of risk for stillbirth subtypes associated with twin gestations among pediatric mothers (10-14 years). Analysis was on twin pregnancies covering the period 1989 to 2000 in the United States. We classified stillbirth as term, preterm, small-for-gestational-age (SGA) or preterm-SGA. We then assessed the risks of these stillbirth subtypes in pediatric mothers using two comparison groups consisting of women aged 15 to 19 years old (adolescent mothers) and 20 to 24 years old (mature mothers). Adjusted risk estimates were by means of hazard ratios generated from a Cox proportional hazards regression model. We adjusted for dependence of observations within twin clusters using the robust sandwich estimator. The rate of stillbirth was highest among pediatric mothers (56/1000), followed by adolescent gravidas (29/1000) and lowest in mature mothers $(20 / 1000 ; p$ for trend <.01). Overall, preterm stillbirth was the most frequent stillbirth phenotype while term stillbirth was the least frequent. Not a single case of term stillbirth was recorded in pediatric mothers. Among pediatric gravidas, the risk for preterm stillbirth was more than tripled (adjusted hazard ratio $[\mathrm{AHR}]=3.4 ; 95 \%$ confidence interval $[\mathrm{Cl}]$ $=2.5-4.6)$, and that of preterm-SGA stillbirth more than doubled $(\mathrm{AHR}=2.6 ; 95 \% \mathrm{Cl}=1.8-3.7)$ that of mature mothers respectively. The $30 \%$ risk elevation for SGA stillbirth among pediatric mothers was not found to be statistically significant (AHR $=1.1 ; 95 \%$ $\mathrm{Cl}=0.3-4.3)$. Pediatric motherhood is a risk factor for stillbirth in twin gestation, especially, preterm and preterm-SGA stillbirth phenotypes. Prevention of stillbirth among this category of mothers should target the period preceding full term.

The birth rate in teen pregnancy remains a significant public health concern in the United States (US) amounting to three times the national average (41.7 per 1000 versus 14.1 per 1000 in 2003; Martin et al., 2005). Previous studies on adverse pregnancy outcomes in pediatric mothers (10-14 years old) have rarely examined the occurrence of stillbirth in this age group; a complication that could perturb future reproductive functions in addition to the psychological ordeal that may leave a long-lasting scar (Satin et al.,
1994). In addition, no data exist on the relationship between stillbirth and pediatric motherhood in twin gestations. To address this gap, we carried out this study to estimate the risk of stillbirth and stillbirth phenotypes among twin deliveries to pediatric mothers.

\section{Materials and Methods}

For this study, we used the vital record data files assembled by the National Center for Health Statistics (NCHS) covering the period 1989 to 2000 (Martin et al., 2000). We selected live-births and fetal deaths of twins between 20 and 44 gestational weeks inclusive. The study group for analysis was pediatric mothers which we defined as gravidas between the ages of 10 and 14 years inclusive. We constructed two comparison groups consisting of women aged 15 to 19 years old (also designated as adolescent mothers) and 20 to 24 years old (also designated as mature mothers).

The main outcome of interest was the occurrence of stillbirth, which was defined as intrauterine fetal death at 20 weeks of gestation or more. Since stillbirth is a heterogeneous concept (Surkan et al., 2004), we further classified the type of stillbirth based on gestational age and fetal growth into term stillbirth (at $\geq 37$ completed gestational weeks), preterm stillbirth (at $<37$ gestational weeks), small-for-gestational-age (SGA) stillbirth (at < 10th percentile of birthweight for gestational age) and preterm-SGA stillbirth comprising of cases who had both conditions concurrently. In classifying infants as SGA, we applied fetal growth curves constructed specifically for twins (Alexander et al., 1998). Gestational age was determined in about $95 \%$ of cases by the date of the last menstrual period. We computed stillbirth rates by dividing the number of stillbirths by the sum of live-births and stillbirths and multiplying by 1000 .

Received 1 February, 2006; accepted 6 July, 2006.

Address for correspondence: Hamisu Salihu, Department of Obstetrics, Gynecology, and Reproductive Sciences, UMDNJ-Robert Wood Johnson Medical School, New Brunswick, NJ 08901-1977, USA. E-mail: hamisu.salihu@gmail.com 


\section{Statistical Analysis}

Although the public data set used for this study indicated whether or not an infant was a twin, matched siblings were not readily identifiable. Since it was imperative to match twin siblings in order to establish and capture the correlations that existed within twin sets, we applied an algorithm that consisted of maternal and infant identifier characteristics to identify matched twin pairs. Infants were considered to be matched twin pairs if the values for the following variables matched: day of the week of birth, month of birth, state and county of birth, maternal age, maternal race/ethnicity, level of maternal education, place of birth of the mother, number of prenatal visits, month prenatal care visits began, and gestational age. The algorithm has been previously validated and found by the authors and other investigators to be accurate (Pollack et al., 2000; Salihu et al., 2003). Using this algorithm, we obtained a perfect 1:1 matching in 314,246 out of the 337,904 individual records $(93.0 \%)$, yielding 157,123 unique sibling pairs. This level of accuracy is similar to that previously reported for matching twin siblings for data sets from the same source (Pollack et al., 2000; Salihu et al., 2003). We restricted our analysis to twins born to mothers aged less than 25 years.

The study group for analysis was pediatric mothers, defined here as gravidas between the ages of 10 and 14 years inclusive. We used the word pediatric and childhood pregnancy interchangeably in this study to refer to pregnancy in 10- to 14-year-old girls. We constructed two comparison groups consisting of women aged 15 to 19 years (also designated as adolescent mothers) and 20 to 24 years old (also designated as mature mothers). We selected live-births and fetal deaths between 20 and 44 gestational weeks inclusive.

We performed crude frequency comparisons and assessed differences in proportions across maternal age
Table 1

Frequency of Preterm and Term Infants Among Twins by Maternal Age Categories (Data Before Matching)

\begin{tabular}{lrccc}
\hline Age & Total & Term & Preterm & $p$ value \\
\hline $10-14$ & 1500 & 30.6 & 69.4 & $<.0001$ \\
$15-19$ & 88,269 & 39.6 & 60.4 & \\
$20-24$ & 248,135 & 45.2 & 54.8 & \\
\hline
\end{tabular}

categories using the chi-square statistic. Dose response or trend patterns were tested for by means of the chi-square test for linear trend. Adjusted estimates were computed using hazard ratios, which were generated through the application of the partial likelihood method described by Cox after testing for nonviolation of the proportionality assumption (Cox, 1972). We confirmed this by plotting the log-negative-log of the Kaplan-Meier estimates of the survival function versus the log of time. The resulting curves were parallel. We also employed the robust sandwich estimator (RSE) to adjust the estimates of the variance of the coefficients in order to account for the correlation among observations within twin sets (Lin \& Wei, 1989).

All tests of hypothesis were two-tailed, with a Type I error rate fixed at $5 \%$. This study was approved by the Institutional Review Board at the University of Alabama at Birmingham, US.

\section{Results}

Ten to 14-year-old mothers had the highest rate of stillbirth $(56 / 1000 ; N=84)$ as compared to those in the age group of 15 to 19 years $(29 / 1000 ; N=2629)$ and 20 to 24 years $(20 / 1000 ; N=4956)$ respectively. Table 1 summarizes the proportion of term and preterm

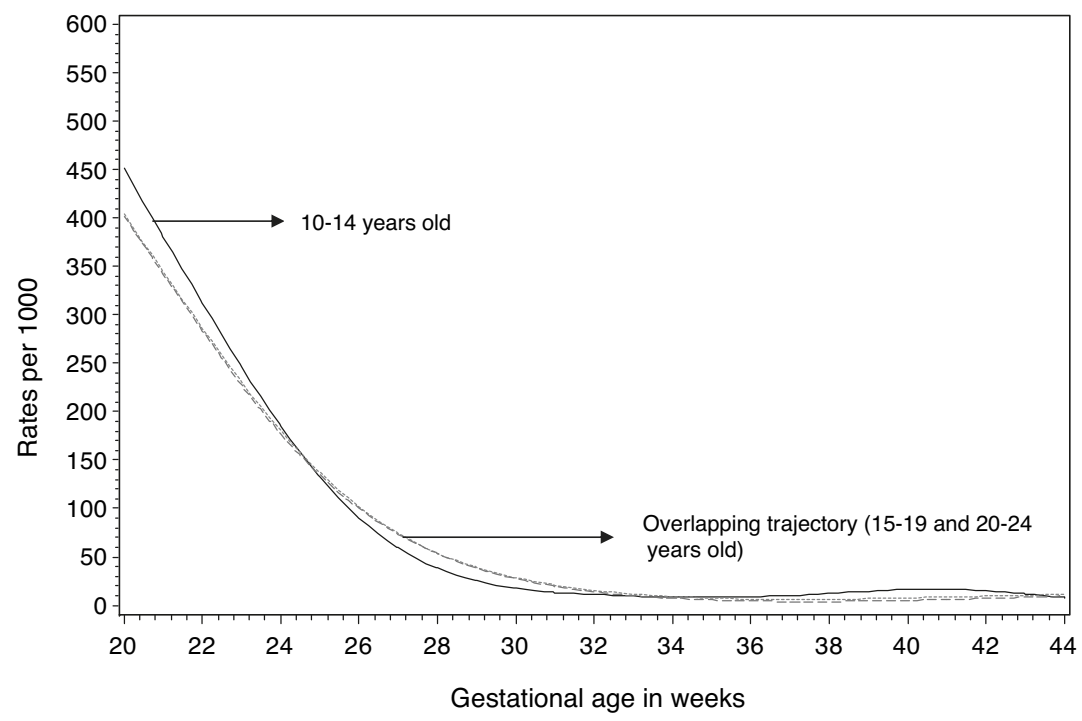

Figure 1

Gestational age-specific stillbirth rates among twins by maternal age.

Note: the rate is expressed as the proportion of individual stillbirths and not individual pregnancies associated with stillbirth. 


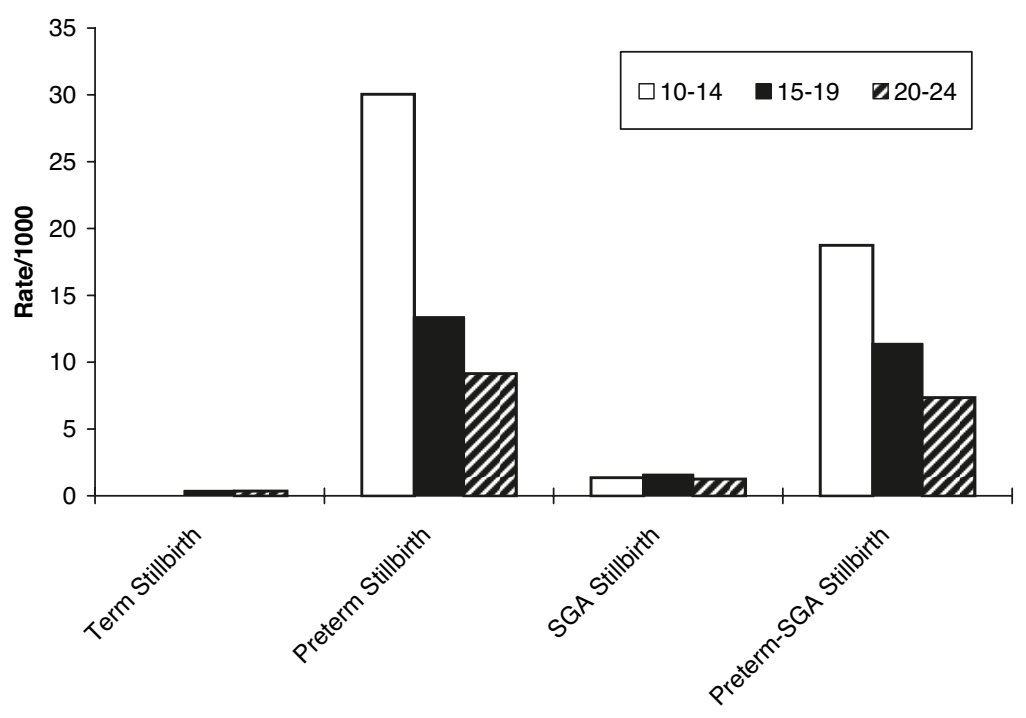

Figure 2

Frequency of stillbirth phenotypes by maternal age group in twins.

twins across the three maternal age groups. Only one third of infants born to pediatric mothers were term, and the frequency of preterm infants diminished with a rise in maternal age.

The gestational age-specific stillbirth rates are depicted in Figure 1. The highest rates of stillbirth were observed among pediatric mothers between the gestational ages of 20 and 24 weeks. A cross-over occurred at around 24 weeks from whence the stillbirth rate for pediatric mothers depreciated appreciably as compared to the two maternal age cohorts until 32 weeks, after which all three trajectories merged until term ( 37 weeks). Thereafter, stillbirth rates among pediatric mothers climbed once again to reproduce the same pattern as that of the initial phase (20-24 weeks). Also noteworthy are the overlapping trajectories for adolescent and mature mothers at almost all gestational weeks.

\section{Table 2}

Adjusted Hazard Ratios for the Association Between Low Maternal Age and Stillbirth Subtypes in Twins

\begin{tabular}{lccc}
\hline & $\begin{array}{c}\text { 10-14 years } \\
\text { AHR (95\% CI) }\end{array}$ & $\begin{array}{c}\text { 15-19 years } \\
\text { AHR }(95 \% \text { CI) }\end{array}$ & $\begin{array}{c}\text { 20-24 years } \\
\text { AHR (95\% CI) }\end{array}$ \\
\hline Term & - & $1.1(0.7-1.6)$ & 1.0 \\
*Preterm & $3.4(2.5-4.6)$ & $1.5(1.4-1.6)$ & 1.0 \\
SGA & $1.1(0.3-4.3)$ & $1.3(1.1-1.6)$ & 1.0 \\
*Preterm-SGA & $2.6(1.8-3.7)$ & $1.6(1.5-1.7)$ & 1.0 \\
\hline
\end{tabular}

Note: $A H R=$ adjusted hazards ratios; $\mathrm{Cl}=$ confidence interval. $\mathrm{SGA}=$ small-for-gestational-age.

Adjusted estimates were obtained after controlling for the confounding effects of race, marital status, parity, prenatal smoking, year of birth, adequacy of prenatal care received, gender of the infant.

${ }^{*} p$ for trend $<.05$
We then examined the incidence of stillbirth by phenotypic categories (preterm, term, SGA and preterm-SGA phenotypes) for twins (Figure 2). Preterm stillbirth was the most frequent subtype irrespective of maternal age while term stillbirth was the least common. Term stillbirth was also most rare among pediatric mothers in comparison to the other maternal age cohorts (not a single term stillbirth was observed in pediatric mothers). On the other hand, crude frequencies of preterm and preterm-SGA stillbirths were greatest among pediatric gravidas.

In Table 2, we present adjusted relative risk estimates for the association between low maternal age and stillbirth subtypes. It is apparent that the hazard ratio for term stillbirth among pediatric mothers could not be computed as there were no cases of term stillbirth recorded among twins born to mothers in this age category within the study period. Among preterm and preterm-SGA infants, low maternal age appeared to be a risk factor for stillbirth. Pediatric gravidas bore the greatest risks for both preterm and preterm-SGA stillbirth phenotypes, with risk estimates that were more than triple and double those of mature mothers respectively. Whereas adolescent mothers had a 30\% higher likelihood for SGA stillbirth when compared to mature mothers, we did not detect a statistically significant difference between pediatric mothers versus mature gravidas with respect to the occurrence of SGA stillbirth phenotype.

\section{$\overline{\text { Discussion }}$}

We found the risk of stillbirth to be elevated among twins born to pediatric mothers. Twins born to these mothers were mostly at risk for preterm and pretermSGA stillbirth subtypes. The risk for preterm and 
preterm-SGA stillbirth more than tripled and doubled respectively among pediatric gravidas.

One limitation of our study is the inability to provide information on the causes of stillbirth because the relevant data as indicated on birth certificates are not considered reliable. In a study carried out in five states, Kirby observed that about $30 \%$ to $45 \%$ of reported causes of stillbirth from vital records were not valid or useful (Kirby, 1993). Furthermore, NCHS does not provide underlying cause of death on its public-use data files. Thus, we did not assess causes of stillbirth to avoid providing erroneous results. Similarly, we were unable to account for the contribution of shorter interpregnancy interval to the occurrence of stillbirth, as the dataset for this study did not provide this specific information.

The risk of stillbirth has been estimated to increase two- to tenfold in women with history of stillbirth (Greenwood et al., 1994; Samueloff et al., 1993), which could have influenced our results as mothers in our comparison groups were more likely to have had a past pregnancy experience, and therefore, stillbirth. Thus, the computed relative risks of stillbirth in pediatric mothers reported in this study were probably underestimated. Unfortunately, specific information on previous fetal loss was also not available in our dataset. The use of the fetuses-at-risk approach would have provided more robust estimates of stillbirth trajectory. However, we were unable to do this because of the paucity of number of stillbirth cases (occasionally zero) in several gestational age categories in pediatric mothers. This limitation also precludes the provision of reliable estimates of standard deviation along the trajectories.

Certain unique features of this study deserve to be highlighted. Very little is known about childhood pregnancy and stillbirth at the population level. Our analysis based on population-based data has the merit of minimizing biases due to selection as well as being more generalizable than otherwise. This is also the largest study to date analyzing the association between this category of mothers and stillbirth, and has sufficient power to show any linkage between the two, if one exists. Additionally, we are unaware of any previously published study examining similar association for twin pregnancies in these mothers and subsequent risk of stillbirth. This new information will potentially improve our understanding of the link between pediatric motherhood and the risk of fetal demise, especially in twins.

\section{Acknowledgment}

This work was supported through a Young Clinical Scientist Award to Dr. Hamisu Salihu by the Flight Attendant Medical Research Institute (FAMRI).

\section{References}

Alexander, G. R., Kogan, M., Martin, J., \& Papiernik, E. (1998). What are the fetal growth patterns of singletons, twins and triplets in the United States? Clinics in Obstetrics and Gynecology, 41, 115-125.

Cox, D. R. (1972). Regression models and life tables. Journal of the Royal Statistical Society, B34, 187-220.

Greenwood, R., Samms-Vaughan, M., Golding, J., \& Ashley, D. (1994). Past obstetric history and risk of perinatal death in Jamaica. Paediatric and Perinatal Epidemiology, 8(Suppl. 1), 40-53.

Kirby, R. S. (1993). The coding of underlying cause of death from fetal death certificates: Issues and policy considerations. American Journal of Public Health, 83, 1088-1091.

Lin, D. Y., \& Wei, L. J. (1989). The robust inference for the Cox proportional hazards model. Journal of the American Statistical Association, 84, 1074-1078.

Martin, J., Curtin, S., Saulnier, M., \& Mousavi, J. (2000). Development of the matched multiple birth file. In 1995-1997 Matched multiple birth dataset. Hyattsville, MD: National Center for Health Statistics.

Martin, J. A., Kochanek, K. D., Strobino, D. M., Guyer, B., \& MacDorman, M. F. (2005). Annual summary of vital statistics - 2003. Pediatrics, 115, 619-634.

Pollack, H., Lantz, P. M., \& Frohna, J. G. (2000). Maternal smoking and adverse birth outcomes among singletons and twins. American Journal of Public Health, 90, 395-400.

Salihu, H. M., Alexander, M. R., Shumpert, N. M., Pierre-Louis, B. J., \& Alexander, G. R. (2003). Infant mortality among twins born to teenagers in the United States: Black-White disparity. Journal of Reproductive Medicine, 48, 257-267.

Samueloff, A., Xenakis, E. M., Berkus, M. D., Huff, R. W., \& Langer, O. (1993). Recurrent stillbirth: Significance and characteristics. Journal of Reproductive Medicine, 38, 883-886.

Satin, A. J., Leveno, K. J., Sherman, M. L., Reedy, N. J., Lowe, T. W., \& McIntire, D. D. (1994). Maternal youth and pregnancy outcomes: Middle school versus high school age groups compared with women beyond the teen years. American Journal of Obstetrics and Gynecology, 177, 184-187.

Surkan, P. J., Stephansson, O., Dickman, P. W., \& Cnattingius, S. (2004). Previous preterm and smallfor-gestational age births and the subsequent risk of stillbirth. New England Journal of Medicine, 350, 777-785. 